\title{
Inheritance of Oryza sativa endornavirus in F1 and F2 hybrids between japonica and indica rice
}

\author{
Hideki Horiuchi ${ }^{\dagger}$, Hiromitsu Moriyama ${ }^{\ddagger}$ and Toshiyuki Fukuhara* \\ Department of Applied Biological Sciences, Faculty of Agriculture, Tokyo University \\ of Agriculture and Technology, Saiwaicho 3-5-8, \\ Fuchu, Tokyo 183-8509, Japan
}

(Received 2 March 2003, accepted 20 May 2003)

\begin{abstract}
We have found a $14 \mathrm{kbp}$ double-stranded RNA (dsRNA) in many cultivars of japonica rice (Oryza sativa L.) but not in any cultivars of indica rice. This dsRNA is an RNA replicon with plasmid-like properties and is proposed to be a novel dsRNA virus, Oryza sativa endornavirus (OSV). Reciprocal crosses between the OSV-carrier japonica variety (Nipponbare) and the OSV-free indica variety (IR 26 or Kasalath) were performed to investigate whether OSV can be transmitted to F1 hybrids. When IR 26 and Nipponbare were used, efficient transmission of OSV from ova (93\%) and pollen (89\%) was observed. When Kasalath and Nipponbare were used, the OSV transmission efficiency to F1 progeny was $68 \%$ from ova and $20 \%$ from pollen. The transmission of OSV to F2 progeny plants was also complicated, showing non-Mendelian inheritance. These results suggest that the dsRNA replicon (OSV) is unstable in indica rice plants.
\end{abstract}

Key words: dsRNA, endornavirus, inheritance, rice

\section{INTRODUCTION}

Rice (Oryza sativa L.) is not only an important food crop but also a model plant. It has been generally accepted that Asian cultivated rice (O. sativa) is divided into indica and japonica subspecies. We have found a large doublestranded RNA (dsRNA) of about $14 \mathrm{kbp}$ in many cultivars of japonica rice, such as Nipponbare, but not in any cultivars of indica rice (Fukuhara et al. 1993; Fukuhara, 1999). We have characterized the dsRNA in the Nipponbare cultivar by biochemical, genetic and molecular biology techniques.

We have found both dsRNA-carrier plants and dsRNAfree plants in the Nipponbare cultivar, and the plants are not visually distinguishable (Moriyama et al. 1996). Therefore, this dsRNA may not affect the rice host. This dsRNA occurs in every tissue and at every developmental stage and is maintained at an almost constant concentration (100 copies/cell) in most tissues, but the copy number increases more than 10-fold in pollen grains (Moriyama et al. 1999a). Although the dsRNA is found in the cytoplasm of host cells, it is transmitted very efficiently (at greater than $98 \%$ ) to progeny plants via pollen and ova in

\footnotetext{
Edited by Yoshio Sano

* Corresponding author. E-mail: fuku@cc.tuat.ac.jp

$\dagger$ Present address: Corporate Research and Development Center, Toshiba Corporation, Komukai 1, Toshiba-cho, Saiwai-ku, Kawasaki 212-8582, Japan.

¥ Toray Industries Inc., Minato-ku, Nagoya 455-8502, Japan.
}

the cross between dsRNA-carrier plants and dsRNA-free plants of the Nipponbare cultivar (Moriyama et al. 1996). Because horizontal transmission of the dsRNA has never been proven, crosses of host plants are a more likely opportunity for dsRNA propagation. An increase in copy number only in pollen grains must be a reasonable strategy for propagation of the dsRNA to progeny despite its cytoplasmic localization. Therefore, the rice dsRNA has some intriguing plasmid-like properties that differ from those of conventional RNA viruses (Fukuhara 1999).

The entire genomic sequence (13,952 nucleotides) of the dsRNA from Nipponbare has been determined (Moriyama et al. 1995). The conserved motifs of RNA-dependent RNA polymerase (RdRp) and RNA helicase (Hel) are found within the one long (13,716 nt) open reading frame (Moriyama et al. 1995). RdRp activity was detected biochemically in crude microsomal fractions from cultured cells of the dsRNA-carrier (Horiuchi et al. 2001). Therefore, this dsRNA is an RNA replicon with plasmid-like properties. However, phylogenetic analyses of the RdRp and Hel motifs of the rice dsRNA indicate that it belongs to an alpha-like supergroup of RNA viruses (Gibbs et al. 2000). We have proposed that the rice dsRNA, Oryza sativa endornavirus (OSV), is a member of a new viral genus (Endornavirus) and family (Endoviridae) (Gibbs et al. 2000).

The results of reciprocal crosses between OSV-carrier and OSV-free japonica variety Nipponbare revealed that 
OSV is efficiently transmitted to the next generation via both pollen and ova (more than 98\%); OSV has been found in many cultivars of japonica rice but not in any strains of indica rice (Fukuhara et al. 1993; Moriyama et al. 1996; Fukuhara 1999). In this study, to investigate whether indica rice varieties can maintain OSV, reciprocal crosses between OSV-free indica rice (IR 26 or Kasalath) and OSV-carrier japonica rice (Nipponbare) were carried out. We report here a unique inheritance mode of the plasmid-like dsRNA replicon (OSV) in rice plants and discuss the possibility of a host factor being involved in OSV replication and maintenance.

\section{MATERIALS AND METHODS}

Detection of the dsRNA in hybrid rice plants. About $3 \mathrm{~g}$ of 14-day-old seedlings or mature leaves of rice plants were pulverized in a mortar after freezing in liquid nitrogen. Intact nucleic acids were extracted by the SDS-phenol method (Fukuhara et al. 1993). In hybrid rice, the dsRNA was isolated by treatment with $50 \mathrm{mg} / \mathrm{ml}$ DNase I in $20 \mathrm{mM} \mathrm{MgCl}_{2}, 300 \mathrm{mM} \mathrm{NaCl}$ and $30 \mathrm{mM}$ sodium citrate for $15 \mathrm{~min}$ at room temperature. The dsRNA samples derived from $50 \mathrm{mg}$ of seedlings (fresh weight) were subjected to electrophoresis on $0.8 \%$ agarose gels containing ethidium bromide $(500 \mathrm{ng} / \mathrm{ml})$. Northern analyses with cDNA probes located 7,372 to $10,084 \mathrm{nt}$ from the 5'-end of the coding strand of the rice dsRNA were also used for detecting the dsRNA in hybrid rice (Fig. 1; Moriyama et al. 1996; 1999b).

Crossing between japonica and indica rice. Three rice cultivars, Nipponbare (japonica), IR 26 (indica) and Kasalath (indica), were used in crossing experiments. Rice plants were grown in a greenhouse at $28^{\circ} \mathrm{C}$. Pollen grains of recipient plants were killed by exposure to water at $43^{\circ} \mathrm{C}$ for $7 \mathrm{~min}$. The success of crossing experiments

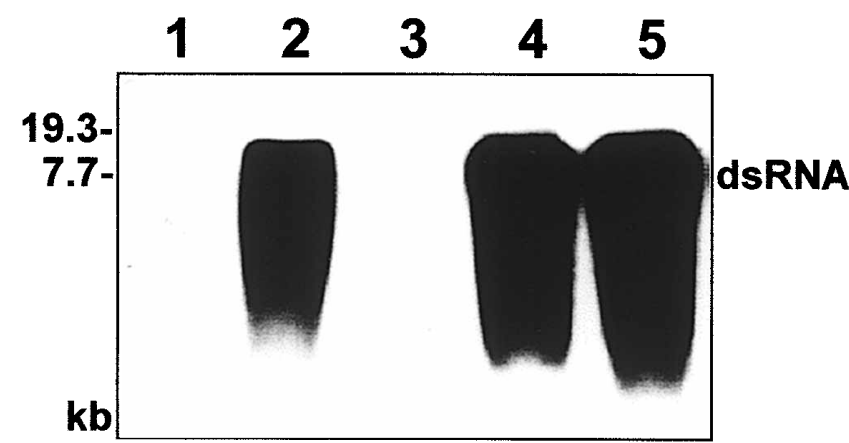

Fig. 1. Detection of the dsRNA in parental and F1 plants. Northern hybridization analysis of dsRNA isolated from $50 \mathrm{mg}$ of leaves from the indica cultivar IR 26 (lane 1), the japonica cultivar Nipponbare (lane 2) and F1 plants between IR 26 (maternal parent) and Nipponbare (paternal parent) (lanes 35). The $14 \mathrm{~kb}$ dsRNA was not detected in the indica cultivar IR 26 (lane 1) and one of F1 plants (lane 3).
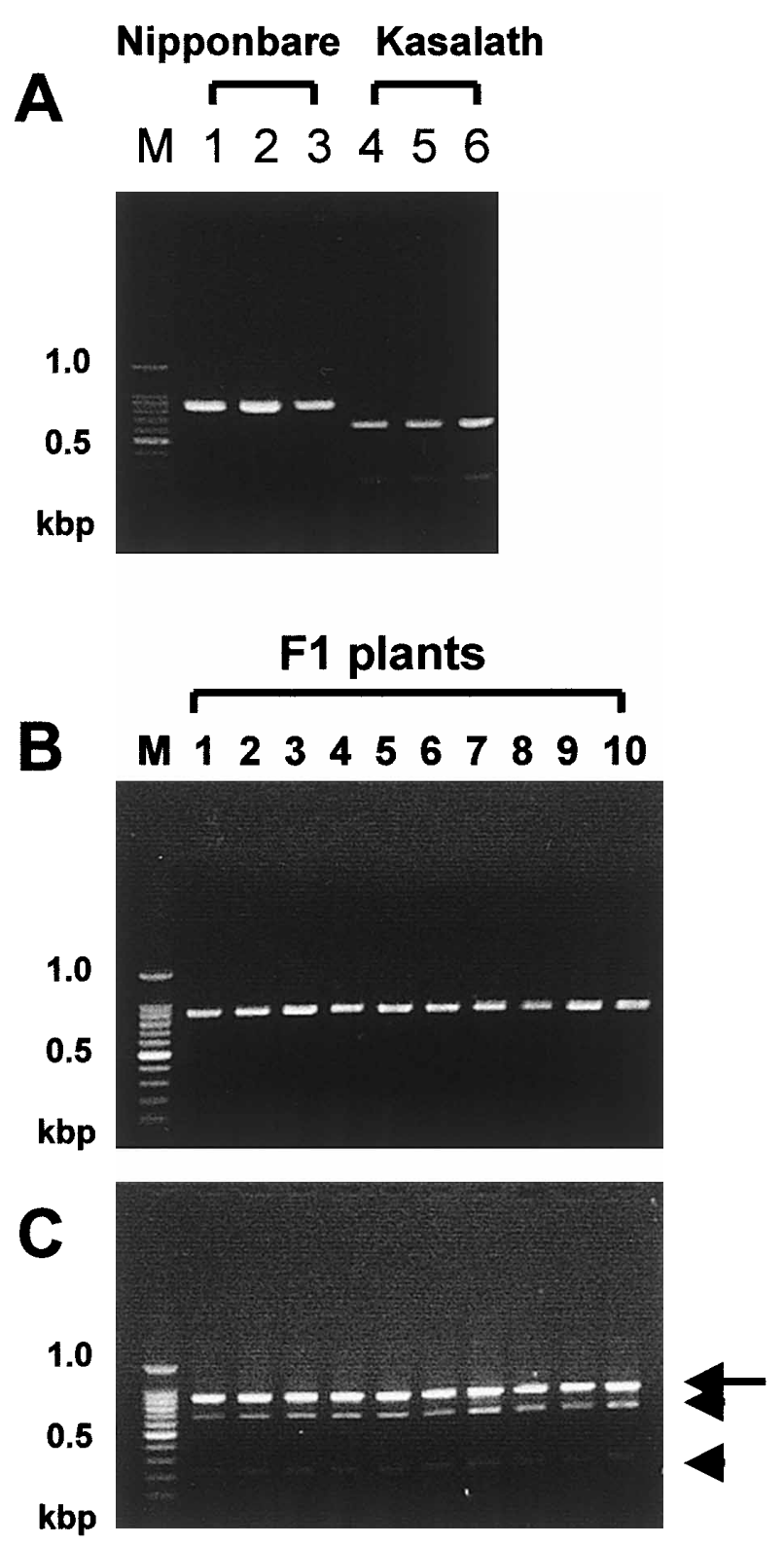

Fig. 2. PCR-RFLP analysis of F1 plants from a cross between the japonica cultivar Nipponbare and the indica cultivar Kasalath. F1 hybrids between Nipponbare and Kasalath were confirmed by the PCR-RFLP analysis. PCR primers were designed for the C-166 locus of chromosome 8 and PCR products were digested by the restriction endonuclease DraI. (A) Agarose gel electrophoresis of PCR products from three individual parental plants (Nipponbare and Kasalath) treated with DraI. Because the PCR product from Kasalath genome DNA contains one DraI site, the $900 \mathrm{bp}$ PCR product was divided into 650 and 250 bp DNA fragments by DraI digestion. On the other hand, no DraI site exists on the corresponding region of the Nipponbare genome. Lanes 1-3, Nipponbare; lanes 4-6, Kasalath. (B) PCR products from genomic DNA of F1 plants. (C) PCR products treated with DraI. PCR products shown in (B) were completely digested with DraI. Each sample contains three DNA fragments: $900 \mathrm{bp}$ from Nipponbare genome (an arrow), and 650 and $250 \mathrm{bp}$ from the Kasalath genome (two arrowheads). The result indicates that all 10 plants were F1 hybrids. Lane M, DNA size markers (100 bp ladder), and lanes 1-10, individual F1 plants. 
Table 1. Transmission of OSV to F1 hybrids between japonica and indica rice

\begin{tabular}{ccccccc}
\hline \hline $\begin{array}{c}\text { Recipient } \\
(\text { + })\end{array}$ & $\begin{array}{c}\text { Pollen donor } \\
\left(\boldsymbol{\sigma}^{\boldsymbol{\pi}}\right)\end{array}$ & Type & $\begin{array}{c}\text { OSV (dsRNA) } \\
(+)^{\mathrm{a}}\end{array}$ & $\begin{array}{c}\text { Total } \\
(-)^{\mathrm{a}}\end{array}$ & $\begin{array}{c}\text { Rate of } \\
\text { Transmission }(\%)\end{array}$ \\
\hline Nipponbare (+)a & IR 26 (-)a & F1 & 14 & 1 & 15 & 93 \\
IR 26 (-) & Nipponbare (+) & F1 & 16 & 2 & 18 & 89 \\
Nipponbare (+) & Kasalath (-) & F1 & $13^{\mathrm{b}}$ & 6 & 19 & 68 \\
Kasalath (-) & Nipponbare (+) & F1 & 3 & 12 & 15 & 20 \\
\hline
\end{tabular}

The Nipponbare cultivar belongs to japonica rice and contains OSV. IR 26 and Kasalath belong to indica rice and do not contain OSV.

a (+) and (-) indicate OSV-carrier and OSV-free plants, respectively.

${ }^{\mathrm{b}}$ The OSV copy number decreased in two F1 plants among 13 OSV-containing F1 plants.

was confirmed by restriction fragment length polymorphism analysis of the PCR products (PCR-RFLP) and by observation of the phenotypes of $\mathrm{F} 1$ plants (for example, the color and shape of seeds, and the height and flowering time of plants; data not shown).

PCR-RFLP analysis. Success of crossing between Nipponbare (japonica) and Kasalath (indica) was confirmed by PCR-RFLP. Oligonucleotides (5'-ATGTCAAACAGTTGCACCAG-3' and 5'AATGAGGAGAATAGGGTTGC-3') derived from the $\mathrm{C}-166$ locus on chromosome 8 of rice were used as primers for PCR (Harushima et al. 2002). Genomic DNA templates for PCR were extracted by the SDS-phenol method, and then treated with RNase A at 50 $\mathrm{mg} / \mathrm{ml}$ overnight at $37^{\circ} \mathrm{C}$ in Tris- $\mathrm{HCl}$ buffer $(\mathrm{pH} 7.0)$. After amplification $\left(94^{\circ} \mathrm{C}\right.$ for 2 min followed by 35 cycles of $94^{\circ} \mathrm{C}$ for $1 \mathrm{~min}, 54^{\circ} \mathrm{C}$ for $2 \mathrm{~min}$ and $72^{\circ} \mathrm{C}$ for $1 \mathrm{~min}$, and $72^{\circ} \mathrm{C}$ for $15 \mathrm{~min}$ ), PCR products were completely digested with DraI for RFLP analysis (Fig. 2).

\section{RESULTS}

Indica rice varieties do not contain the dsRNA replicon (OSV). The $14 \mathrm{kbp}$ dsRNA has been found in many strains of temperate and tropical japonica rice, but not in any strains of indica rice (Fukuhara et al. 1993; Fukuhara 1999; Moriyama et al. 1999b). We surveyed 11 cultivars of indica rice to determine whether they contain the dsRNA, but no strains of indica rice examined, including IR 26 and Kasalath, contained the dsRNA (data not shown). This dsRNA is not transcribed from rice genomic DNA but replicates autonomously in the cytoplasm of rice cells by its own replicase (RNA-dependent RNA polymerase) (Horiuchi et al. 2001). This dsRNA has some plasmid-like properties but it is classified as a virus (Oryza sativa endornavirus; OSV) (Gibbs et al. 2000). We surmised that indica rice might not have a system for maintenance of this dsRNA replicon (OSV). Crossing experiments between OSV-carrier japonica rice and OSV-free indica rice were carried out to investigate the maintenance of OSV.
Inheritance of OSV in F1 hybrids. Reciprocal crosses between OSV-carrier japonica variety (Nipponbare) and OSV-free indica variety (IR 26 or Kasalath) were performed in order to introduce OSV into F1 progeny plants via pollen or ova and to investigate whether F1 hybrid plants can maintain OSV. Nipponbare and Kasalath varieties are representative varieties of japonica and indica rice, respectively, both of which have been selected for the rice genome project (Harushima et al. 1998).

When IR 26 (indica rice) was used as a parent, efficient transmission of OSV from pollen (89\%) and ova (93\%) was observed in F1 plants (Table 1). In contrast to IR 26, when Kasalath was used as the indica parent, the OSV transmission efficiency to F1 progeny was low. When Kasalath was the paternal parent and Nipponbare was the maternal parent, the efficiency of OSV transmission to $\mathrm{F} 1$ progeny was $68 \%$ (Table 1). When Kasalath was the maternal parent and Nipponbare was the paternal parent, the efficiency of OSV transmission to F1 progeny

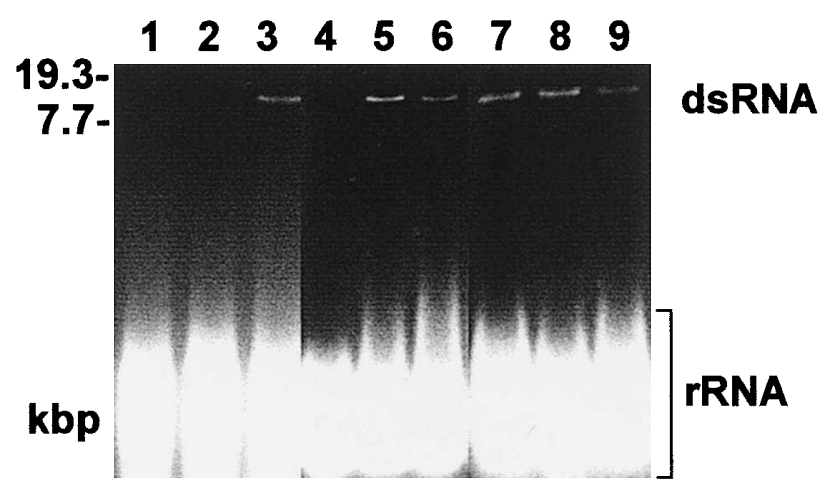

Fig. 3. Comparison of dsRNA copy number between parental and F1 plants. Agarose gel electrophoresis of dsRNAs isolated from $50 \mathrm{mg}$ of leaves from the indica cultivar IR 26 (lane 1), the indica cultivar Kasalath (lane 2), the japonica cultivar Nipponbare (lane 3), F1 plants between Nipponbare (maternal parent) and IR 26 (paternal parent) (lanes 4-6) and F1 plants between IR 26 (maternal parent) and Nipponbare (paternal parent) (lanes 7-9). Althoguh the dsRNA was not detected in one of F1 plants (lane 4), no significant difference in dsRNA copy number between the japonica cultivar Nipponbare (lane 3) and OSV-containing F1 plants (lanes 5-9) was found. 
was only $20 \%$ (Table 1). The success of crossing between Nipponbare and Kasalath was confirmed by PCR-RFLP (Fig. 2). All F1 hybrids examined contained both Nipponbare and Kasalath genomes. No significant difference in OSV copy number between OSV-containing F1 plants and parental japonica rice (100 copies/cell) was found by agarose gel electrophoresis (Fig. 3), except a decreased copy number of OSV in two out of 13 OSV-containing F1 plants resulting from crosses between a maternal Nipponbare and paternal Kasalath (Table 1).

OSV inheritance in F2 progeny plants. To investi- gate OSV inheritance in F2 progeny, two panicles were randomly collected from each $\mathrm{F} 1$ plant, and then 13 seeds from each panicle were sown (Table 2). All plants that germinated were analyzed for the presence of the $14 \mathrm{kbp}$ dsRNA (OSV). The OSV transmission rates from both panicles of individual F1 plants to the F2 generation were similar; namely, the transmission rate of panicle (A) was almost the same as that of panicle (B) for each F1 plant (Table 2). However, the OSV transmission efficiency from different $\mathrm{F} 1$ plants to $\mathrm{F} 2$ plants was variable $(0 \%$ to 100\%). None of the F2 plants derived from OSV-free F1 plants harbored the dsRNA.

Table 2. Transmission of OSV to F2 plants from the cross between japonica and indica rice

\begin{tabular}{|c|c|c|c|c|c|c|}
\hline \multirow{2}{*}{$\begin{array}{l}\text { Receipient } \\
\text { ( 우 ) }\end{array}$} & \multirow{2}{*}{$\begin{array}{c}\text { Pollen doner } \\
\left(\sigma^{\star}\right)\end{array}$} & \multirow{2}{*}{$\begin{array}{l}\text { F1 plant } \\
\text { number }\end{array}$} & \multirow[t]{2}{*}{ Panicle $^{b}$} & \multicolumn{2}{|c|}{ OSV (dsRNA) } & \multirow{2}{*}{$\begin{array}{c}\text { Rate of } \\
\text { transmission }(\%)\end{array}$} \\
\hline & & & & $(+)^{\mathrm{a}}$ & $(-)^{\mathrm{a}}$ & \\
\hline \multirow[t]{5}{*}{ Nipponbare $(+)^{\mathrm{a}}$} & IR $26(-)^{\mathrm{a}}$ & $1(-)^{\mathrm{a}}$ & $\mathrm{A}$ & 0 & 8 & 0 \\
\hline & & $2(+)^{\mathrm{a}}$ & $\mathrm{A}$ & 9 & 1 & 90 \\
\hline & & & B & 8 & 2 & 80 \\
\hline & & $3(+)$ & $\mathrm{A}$ & 0 & 7 & 0 \\
\hline & & & B & 0 & 7 & 0 \\
\hline \multirow[t]{5}{*}{ IR $26(-)$} & Nipponbare (+) & $1(-)$ & $\mathrm{A}$ & 0 & 12 & 0 \\
\hline & & $2(+)$ & $\mathrm{A}$ & 0 & 7 & 0 \\
\hline & & & B & 0 & 7 & 0 \\
\hline & & $3(+)$ & $\mathrm{A}$ & 10 & 0 & 100 \\
\hline & & & B & 9 & 0 & 100 \\
\hline \multirow[t]{10}{*}{ Nipponbare (+) } & Kasalath (-) & $1(-)$ & $\mathrm{A}$ & 0 & 12 & 0 \\
\hline & & & B & 0 & 13 & 0 \\
\hline & & $2(+)$ & $\mathrm{A}$ & 7 & 6 & 54 \\
\hline & & & B & 7 & 5 & 58 \\
\hline & & $3(+)$ & $\mathrm{A}$ & 8 & 4 & 67 \\
\hline & & & B & 10 & 3 & 77 \\
\hline & & $4(+)$ & $\mathrm{A}$ & 0 & 13 & 0 \\
\hline & & & B & 0 & 12 & 0 \\
\hline & & $5(+)$ & $\mathrm{A}$ & 0 & 12 & 0 \\
\hline & & & B & 0 & 12 & 0 \\
\hline \multirow[t]{8}{*}{ Kasalath (-) } & Nipponbare (+) & $1(-)$ & $\mathrm{A}$ & 0 & 12 & 0 \\
\hline & & & B & 0 & 12 & 0 \\
\hline & & $2(+)$ & $\mathrm{A}$ & 0 & 12 & 0 \\
\hline & & & B & 0 & 13 & 0 \\
\hline & & $3(+)$ & $\mathrm{A}$ & 0 & 12 & 0 \\
\hline & & & B & 0 & 13 & 0 \\
\hline & & $4(+)$ & $\mathrm{A}$ & 12 & 1 & 92 \\
\hline & & & B & 12 & 1 & 92 \\
\hline
\end{tabular}

The Nipponbare cultivar belongs to japonica rice and contains OSV. IR 26 and Kasalath belong to indica rice and do not contain OSV.

${ }^{\mathrm{a}}(+)$ and (-) indicate OSV-carrier and OSV-free plants, respectively.

${ }^{\mathrm{b}}$ Two panicles (A and B) were randomly collected from each F1 plant; 13 seeds from each panicle were sown, but some seeds did not germinate. All germinated plants were analyzed. 


\section{DISCUSSION}

In our previous report, the results of crossing experiments in the japonica variety Nipponbare between OSVcarrier and OSV-free plants indicate that OSV is transmitted at greater than $98 \%$ efficiency to the next generation via both pollen and ova, and also that the absence of OSV in japonica varieties does not depend on any particular gene in OSV-free plants (Moriyama et al. 1996). All 11 cultivars of indica rice examined lacked OSV. It is not clear why OSV is not found in any indica rice varieties. The inheritance of OSV in F1 and F2 hybrids between indica and japonica was not consistent with Mendel's law, and many F1 and F2 hybrid plants had lost the dsRNA (Tables 1 and 2). Because OSV is an RNA replicon that can replicate autonomously in the host cell's cytoplasm, the OSV transmission efficiency in F1 and F2 plants may depend on its replication efficiency in somatic cells during embryonic development of F1 and F2 plants. Therefore, the results suggest that the dsRNA replicon (OSV) is unstable in indica rice plants. Furthermore, the results suggest that (1) indica rice does not have host factors essential for OSV replication or maintenance or (2) indica rice possesses a mechanism that eliminates OSV from its cells.

The mechanism by which some F1 and F2 plants lose OSV is not clear yet. However, the results of OSV transmission to $\mathrm{F} 1$ hybrids suggest that the $\mathrm{F} 1$ zygote is likely to contain a certain number of OSV that came from either an egg or a sperm cell of japonica rice. If the F1 zygote has host factors for eliminating OSV or does not have enough host factors for maintaining it, the cells in the F1 plant must lose OSV as cell divisions proceed. Once these F1 cells have lost OSV, their descendant F1 cells will never reacquire it.

It is generally accepted that cytoplasmic factors, such as chloroplasts and mitochondria, are completely transmitted to F1 progeny only from the maternal parent in many angiosperms (Birky 1995). However, the transmission rate of OSV to $\mathrm{F} 1$ plants from the maternal japonica (cv. Nipponbare) was not $100 \%$. It was only $68 \%$ when Kasalath was the paternal parent and Nipponbare was the maternal parent (Table 1). On the other hand, when OSV-carrier japonica rice was the paternal parent and OSV-free indica rice was the maternal parent, some of the $\mathrm{F} 1$ plants ( $89 \%$ or $20 \%$, depending on the variety) had OSV (Table 1). Therefore, the inheritance of OSV to F1 hybrids between indica and japonica rice is not consistent with either Mendelian inheritance as nuclear genes or with uniparental inheritance as mitochondrial or chloroplast genes.

It is not clear why the OSV transmission efficiencies differ between the varieties used as the indica parent (Kasalath or IR 26) in crosses. Because these two indica varieties do not have the same genetic background, dif- ferent OSV transmission efficiencies may be expected. The OSV transmission efficiencies in F1 hybrids reported in Table 1 suggest that IR 26 is likely to be genetically closer to japonica rice than Kasalath.

Except for our reports of the inheritance of the rice dsRNA replicon (OSV) (Moriyama et al. 1996; 1999a; 1999b), inheritance of extrachromosomal replicons in plants has never been reported. Because Mendel's law can be applied only to nuclear genes and OSV is an RNA replicon in the cytoplasm, it is not surprising that its inheritance is not consistent with Mendel's law. Because the entire nucleotide sequence of the genomic DNA of japonica rice (cv. Nipponbare) has been published recently, the rice plant (cv. Nipponbare) and the dsRNA replicon (OSV) seem to be a good model system for studying molecular interactions between the RNA replicon and the host plant cell.

We thank Drs. K. Ozawa and M. Yano, National Institute of Agrobiological Resources, Tsukuba, Japan, for help with crossing experiments and for providing information about the C-166 locus. This research was supported in part by Grant-in-Aid for Scientific Research (B) No. 14390019 to T.F. from the Ministry of Education, Science, Sports, Culture and Technology of Japan.

\section{REFERENCES}

Birky, C. W. Jr. (1995) Uniparental inheritance of mitochondrial and chloroplast genes: mechanisms and evolution. Proc. Natl. Acad. Sci. USA 92, 11331-11338.

Fukuhara, T. (1999) Double-stranded RNA in rice. J. Plant Res. 112, 131-138.

Fukuhara, T., Moriyama, H., Pak, J. Y., Hyakutake, T., and Nitta, T. (1993) Enigmatic double-stranded RNA in Japonica rice. Plant Mol. Biol. 21, 1121-1130.

Gibbs, M. J., Koga, K., Moriyama, H., Pfeiffer, P., and Fukuhara, T. (2000) Phylogenetic analysis of some large doublestranded RNA replicons from plants suggests they evolved from a defective single-stranded RNA virus. J. Gen. Virol. 81, 227-233.

Harushima, Y., Yano, M., Shomura, A., Sato, M., Shimano, T., Kuboki, Y., Yamamoto, T., Lin, S. Y., Antonio, B. A., Parco, A., Kajiya, H., Huang, N., Yamamoto, K., Nagamura, Y., Kurata, N., Khush, G. S., and Sasaki, T. (1998) A high-density rice genetic linkage map with 2275 markers using a single F2 population. Genetics 148, 479-494.

Harushima, Y., Nakagahra, M., Yano, M., Sasaki, T., and Kurata, N. (2002) Diverse variation of reproductive barriers in three intraspecific rice crosses. Genetics 160, 313-322.

Horiuchi, H., Udagawa, T., Koga, K., Moriyama, H., and Fukuhara, T. (2001) RNA-dependent RNA polymerase activity associated with endogenous double-stranded RNA in rice. Plant Cell Physiol. 42, 197-203.

Moriyama, H., Nitta, T., and Fukuhara, T. (1995) Doublestranded RNA in rice: a novel RNA replicon in plants. Mol. Gen. Genet. 248, 364-369.

Moriyama, H., Kanaya, K., Wang, J. Z., Nitta, T., and Fukuhara, T. (1996) Stringently and developmentally regulated levels of a cytoplasmic double-stranded RNA and its high efficiency transmission via egg and pollen in rice. Plant Mol. Biol. 31, 713-719. 
Moriyama, H., Horiuchi, H., Koga, R., and Fukuhara, T. (1999a) Molecular characterization of two endogenous doublestranded RNAs in rice and their inheritance by interspecific hybrids. J. Biol. Chem. 274, 6882-6888.

Moriyama, H., Horiuchi, H., Nitta, T., and Fukuhara, T. (1999b)
Unusual in heritance of evolutionarily-related doublestranded RNAs in interspecific hybrid between rice plants Oryza sativa and Oryza rufipogon. Plant Mol. Biol. 39, $1127-1136$ 